\title{
High Average Power Second-harmonic Generation of a CW Erbium Fiber MOPA
}

\author{
T. H. Runcorn, R. T. Murray, and J. R. Taylor, Member, IEEE
}

\begin{abstract}
We report the generation of $28 \mathrm{~W}$ of $780 \mathrm{~nm}$ radiation with near diffraction limited beam quality $\left(\mathrm{M}^{2} \leq \mathbf{1 . 1 5}\right)$ by frequency-doubling a continuous-wave (CW) erbium fiber master oscillator power amplifier (MOPA) system in a periodically poled lithium niobate crystal. The second-harmonic generation conversion efficiency reached $45 \%$ with no rolloff observed, suggesting that further power scaling should be possible with higher fundamental pump powers. The generated second-harmonic had a $3 \mathrm{~dB}$ spectral bandwidth of $0.10 \mathrm{~nm}$. The presented architecture represents a simple and effective route to generating high-brightness radiation around $780 \mathrm{~nm}$.
\end{abstract}

Index Terms-Second harmonic generation (SHG), erbium fiber laser, periodically poled lithium niobate (PPLN).

\section{INTRODUCTION}

$\mathbf{H}$ IGH average-power continuous-wave $(\mathrm{CW})$ radiation around $780 \mathrm{~nm}$ with near diffraction-limited beam quality is required for a variety of scientific applications, such as spectroscopy, laser cooling of atoms [1] and the pumping of alkali gas-vapor lasers [2]. Direct laser emission can be obtained at this wavelength by semiconductor laser diodes and a variety of solid-state gain media but scaling the diffractionlimited average-power of these lasers to multiple tens of Watts remains challenging.

Semiconductor laser diodes are often the preferred option due to their high efficiency and the practical advantages associated with electrical pumping, however, the single-mode average-power is limited by catastrophic optical damage to the output facet. The use of tapered semiconductor gain regions has been used to mitigate this limitation but tapered amplifiers operating at $780 \mathrm{~nm}$ are still restricted to average-powers of only a few Watts [3].

Of the solid-state gain media available in this wavelength region, Ti:Sapphire is by far the most widespread and commercially mature. Argon-ion laser pumped Ti:Sapphire systems have been demonstrated with $\mathrm{CW}$ powers reaching $43 \mathrm{~W}$, but cryogenic cooling of the two laser crystals was required to alleviate thermally induced optical distortions in the laser rods [4]. In addition to the thermal issues associated with the relatively large quantum defect, the power scaling of Ti:Sapphire lasers

Manuscript received December 20, 2016; revised June 6, 2017; accepted July 31, 2017. This work was supported by the Engineering and Physical Sciences Research Council (EP/N009452/1 and EP/K503733/1) and by the European Office of Aerospace Research and Development (FA9550-17-10194).

The authors are with the Femtosecond Optics Group, Department of Physics, Imperial College London, Prince Consort Road, London, SW7 2BW, UK (email: timothy.runcorn07@imperial.ac.uk).

Copyright (c) 2016 IEEE. Personal use of this material is permitted However, permission to use this material for any other purposes must be obtained from the IEEE by sending a request to pubs-permissions@ieee.org. is also hindered by the lack of available high-brightness semiconductor pump diodes [5]. High average-power Ti:Sapphire lasers rely on frequency-doubled solid-state or semiconductor pump lasers, which significantly reduces the overall optical-tooptical efficiency, thereby limiting the power-scaling potential.

The difficulties associated with diode-pumping Ti:Sapphire has fuelled the research effort into alternative solid-state gain media, such as Cr:LiSAF $\left(\mathrm{Cr}^{3+}: \mathrm{LiSrAlF}_{6}\right), \mathrm{Cr}: \mathrm{LiCAF}$ $\left(\mathrm{Cr}^{3+}: \mathrm{LiCaAlF}_{6}\right)$ and Alexandrite $\left(\mathrm{Cr}^{3+}: \mathrm{BeAl}_{2} \mathrm{O}_{4}\right)$ [6]-[8], which can be pumped by available high-brightness semiconductor laser diodes. The superior thermal and mechanical properties of Alexandrite over the colquiriites has enabled the $\mathrm{CW}$ power to be scaled to $26 \mathrm{~W}$ at $759 \mathrm{~nm}$ with multiple transverse modes, pumped by diode bars operating at $639 \mathrm{~nm}$ [8]. The highest reported near diffraction-limited CW power at $780 \mathrm{~nm}$ for Alexandrite is, however, only around the Watt-level [9].

An alternative route to generating radiation around $780 \mathrm{~nm}$ is to frequency-double the output of an Er:fiber laser. Fiber lasers are ideally suited to nonlinear frequency conversion due to their ability to be power-scaled whilst maintaining excellent beam quality. In addition, they are commercially available with very high average powers in compact air-cooled units with high wall-plug efficiencies. Er:fiber lasers have been scaled to CW powers of $297 \mathrm{~W}$ [10], therefore, provided the secondharmonic generation (SHG) can be performed efficiently, the diffraction-limited $\mathrm{CW}$ power of solid-state lasers can be surpassed.

The low peak-power of $\mathrm{CW}$ lasers is the main obstacle to efficient SHG, therefore, external enhancement cavities are typically used to perform efficient frequency-doubling of $\mathrm{CW}$ lasers. The use of an external cavity adds significant complexity, requiring a single-frequency pump laser and a cavity length locking mechanism, as well as sensitive optical alignment. An alternative approach is to use a nonlinear crystal with a high nonlinear coefficient in a simple, singlepass optical setup. The use of quasi phase-matching (QPM) with periodically poled lithium niobate (PPLN) enables access to the largest $d_{33}$ component of the second-order nonlinear susceptibility tensor $\left[\chi^{(2)}\right]$ through type-0 (all waves extraordinarily polarized) phase-matching. Typical PPLN devices have $\mathrm{d}_{\text {eff }} \sim 16 \mathrm{pm} / \mathrm{V}$ [11] and are available in lengths up to $80 \mathrm{~mm}$, facilitating efficient SHG of CW laser sources.

To date, the highest reported average-power for a frequencydoubled CW Er:fiber laser is $11 \mathrm{~W}$ with an SHG conversion efficiency of $36 \%$ using a $40 \mathrm{~mm}$ long $5 \mathrm{~mol} \% \mathrm{MgO}$-doped PPLN crystal [1]. In the quasi-CW regime, a peak-power of $34 \mathrm{~W}$ with a conversion efficiency of $52 \%$ using a single 


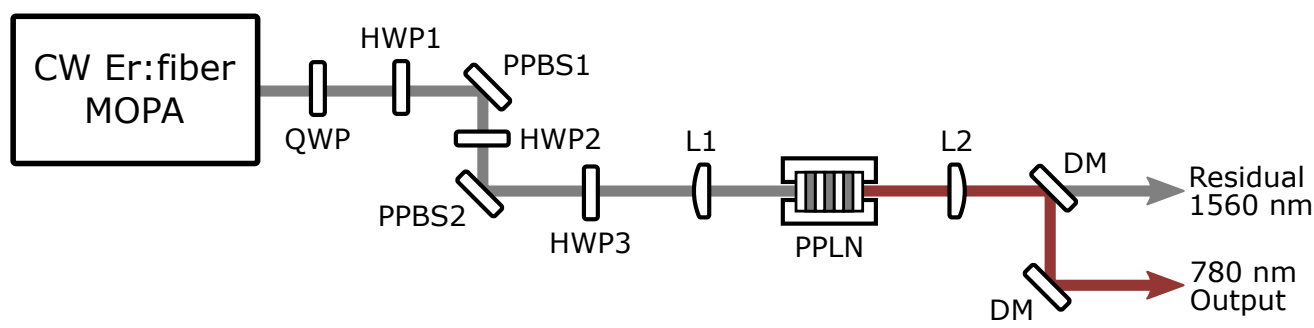

Fig. 1. Schematic of the frequency-doubled Er:fiber MOPA system. QWP, quarter waveplate; HWP, half waveplate; PPBS, polarizing plate beamsplitter; L1, focusing lens; L2, collimating lens; DM, dichroic mirror; PPLN, periodically poled lithium niobate.

PPLN crystal and a peak-power of $43 \mathrm{~W}$ with a conversion efficiency of $66 \%$ using a cascade of two PPLN crystals has been demonstrated [12]. At $775 \mathrm{~nm}$, an average-power of $41 \mathrm{~W}$ has been generated in a periodically poled potassium titanyl phosphate (PPKTP) crystal with a conversion efficiency of $72 \%$ using nanosecond pulses [13]. These results are encouraging for high average-power CW SHG in periodically poled crystals but to date, the highest reported $\mathrm{CW}$ power is $19 \mathrm{~W}$ at $532 \mathrm{~nm}$ using periodically poled lithium tantalate (PPLT) [14], [15].

In this manuscript we report the generation of $28 \mathrm{~W}$ of near diffraction limited $\left(\mathrm{M}^{2} \leq 1.15\right) \mathrm{CW}$ light at $780 \mathrm{~nm}$ by frequency-doubling an Er:fiber master oscillator power amplifier (MOPA) system in a PPLN crystal. To the best of our knowledge, this is the highest CW SHG average-power generated in a periodically poled crystal. The SHG conversion efficiency reached $45 \%$ at the highest fundamental power without roll-off, indicating that further power scaling could be achieved with higher fundamental powers.

\section{EXPERIMENTAL SETUP}

The simple, single-pass SHG experimental setup is shown in Fig. 1. A CW Er:fiber MOPA system operating at $1560 \mathrm{~nm}$ was used as the fundamental light source, which utilized a tunable external fiber Bragg grating cavity semiconductor diode as the master oscillator. The use of isotropic fiber in both the master oscillator cavity and the subsequent amplifier stages resulted in a randomly polarized MOPA output that varied in time. A combination of a quarter waveplate (QWP) and a half waveplate (HWP1) was used to linearize the output polarization and align it to the reflection axis (s-polarized) of the first polarizing plate beamsplitter (PPBS1). A second half waveplate (HWP2) was used in combination with the second polarizing plate beamsplitter (PPBS2) as a variable power control. This enabled the fundamental power to be varied without affecting other parameters of the beam, for example the spectral characteristics, beam quality and beam pointing, which would have otherwise changed if the pump power of the Er:fiber MOPA was varied.

A third half waveplate (HWP3) was used after PPBS2 to align the polarized pump light to the extraordinary axis of the PPLN crystal, in order to access the $d_{33}$ nonlinear component via type- 0 phasematching. A $30 \mathrm{~mm}$ long, $5 \mathrm{~mol} . \%$ MgO-doped PPLN crystal (HC Photonics) with a $3.4 \times 1 \mathrm{~mm}^{2}$ aperture was used for SHG, which was anti-reflection (AR) coated on both input and output faces $(780 \mathrm{~nm} \mathrm{R}<1 \%$, $1560 \mathrm{~nm} \mathrm{R}<0.5 \%)$. The PPLN crystal was poled with a single $19.48 \mu \mathrm{m}$ pitch grating, resulting in a $3 \mathrm{~dB}$ spectral acceptance bandwidth of $0.41 \mathrm{~nm}$ for a fundamental wavelength of $1560 \mathrm{~nm}$. The crystal was mounted in a copper oven held at $84.7^{\circ} \mathrm{C}$ to ensure phasematching of the $1560 \mathrm{~nm}$ light and indium foil was used between the crystal and the copper oven to increase the thermal conductivity of the interface.

Following an empirical optimization of the focusing parameter, an AR coated $\mathrm{f}=150 \mathrm{~mm}$ plano-convex lens (L1) was used to focus the fundamental light into the center of the PPLN crystal. The focused spot diameter $\left(1 / \mathrm{e}^{2}\right)$ was $73 \mu \mathrm{m}$ with a measured Rayleigh range in air of $3.1 \mathrm{~mm}$, corresponding to a focusing parameter of $\xi=L / b=2.3$, where $L$ is the crystal length and $b$ is the confocal parameter. The measured beam quality factors of the fundamental were $\mathrm{M}^{2}=1.23$ and $\mathrm{M}^{2}=1.18$ in the vertical and horizontal axes, respectively. To ensure accurate power measurements, the power data was taken using a shortpass dichroic mirror immediately after the PPLN crystal, with a measured $\mathrm{T}=95 \%$ at $780 \mathrm{~nm}$ and $\mathrm{R}=1.7 \%$ at $1560 \mathrm{~nm}$. For the second-harmonic (SH) beam characterization, the generated $\mathrm{SH}$ was collimated using a AR coated $f=150 \mathrm{~mm}$ lens and the fundamental separated by reflecting off two longpass dichroic mirrors (Thorlabs DMLP950).

\section{RESULTS AND DISCUSSION}

The generated SH power (red points), corrected for the shortpass reflection of the $\mathrm{SH}$ and transmission of the fundamental, as a function of fundamental pump power is shown in Fig. 2. A maximum SH power of $28.2 \mathrm{~W}$ was obtained for a fundamental power of $62.2 \mathrm{~W}$, corresponding to a conversion efficiency of $45 \%$. For focussed Gaussian beams, the SHG conversion efficiency including the effects of pump depletion at the optimum phase-mismatch is well approximated by [16]:

$$
\eta=\tanh ^{2}\left[\sqrt{\frac{16 \pi^{2} d_{\mathrm{eff}}^{2}}{n(2 \omega) n(\omega) \epsilon_{0} c \lambda^{3}} P_{\omega} L h}\right]
$$

where $d_{\mathrm{eff}}$ is the QPM effective nonlinear coefficient, $n(2 \omega)$ and $n(\omega)$ are the refractive indices at the $\mathrm{SH}$ and fundamental frequencies, $\lambda$ is the fundamental wavelength, $P_{\omega}$ is the fundamental peak power, $L$ is the crystal length and $h$ is the Boyd-Kleinman $h$-factor [17].

Excellent agreement between the measured SHG conversion efficiency (black points) and the expected $\tanh ^{2}\left[\sqrt{P_{\omega}}\right]$ 


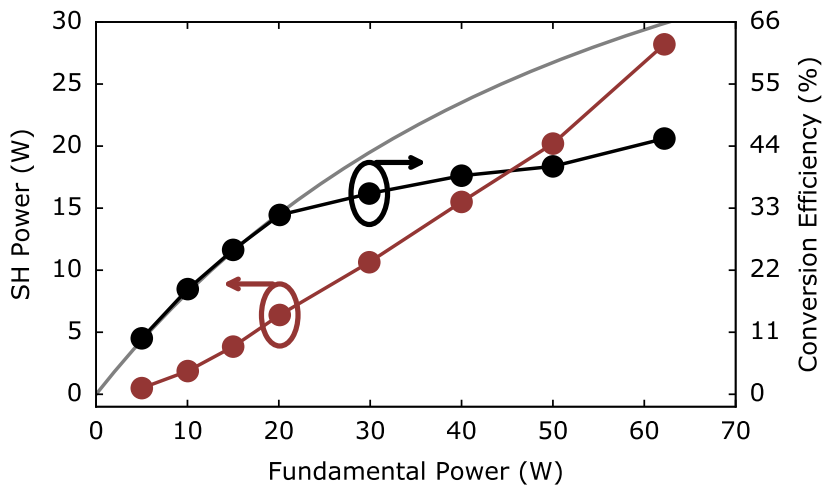

Fig. 2. Generated second-harmonic (SH) average power at $780 \mathrm{~nm}$ (red points) and SHG conversion efficiency (black points) as a function of fundamental $(1560 \mathrm{~nm})$ average power, connecting lines are a guide for the eye. The SHG conversion efficiency is fitted with a tanh ${ }^{2}$ curve, according to Eqn. 1 (grey line).

relationship (grey line) was obtained for fundamental powers up to $20 \mathrm{~W}$ (Fig. 2). For fundamental powers $>20 \mathrm{~W}$, the rate of the SHG conversion efficiency increase with pump power was significantly lower than expected from theory. We attribute this decline in the rate of the SHG conversion efficiency increase to the onset of thermal issues caused by the absorption of the generated SH in the PPLN crystal. The spatially inhomogeneous nature of the heat load generated by the absorption of the $\mathrm{SH}$ leads to local temperature variations in the crystal that disrupt the QPM, thereby reducing the SHG conversion efficiency [18].

The temporal characteristics of the randomly polarized output of the Er:fiber MOPA are shown in Fig. 3(a), where the average power after PPBS2 with HWP2 set for maximum reflected power was recorded for 240 mins following turnon from a cold start. The degree of polarization (DOP) of the Er:fiber MOPA periodically varied from completely unpolarized $[0 \mathrm{~dB}$ polarization extinction ratio (PER), $\sim 30 \mathrm{~W}$ polarized power] to a PER $>13 \mathrm{~dB}$, with a period of oscillation that increased with time [Fig. 3(a)]. It was observed that the orientation of the polarization ellipse and the ellipticity changed between the maxima and minima of the DOP, but remained constant when at the maxima (i.e. no adjustment of the QWP/HWP1 was necessary).

This behaviour was non-ideal in light of the polarizationsensitive nature of the SHG process, however, the Er:fiber MOPA delivered $>62 \mathrm{~W}$ of polarized power for periods exceeding 60 minutes [Fig. 3(a)], which enabled the SHG to be investigated. It is worth noting that this shortcoming could easily be rectified with the use of a polarized Er:fibre MOPA, which is the subject of future work. The power stability of the SH during a maxima of the DOP of the Er:fiber MOPA was excellent, with peak-to-peak fluctuations of $<1 \%$ over a period of 40 minutes at an average-power of $28 \mathrm{~W}$ [Fig. 3(b)].

To characterize the beam quality of the $\mathrm{SH}$, the collimated $780 \mathrm{~nm}$ beam was focused using an AR coated $\mathrm{f}=120 \mathrm{~mm}$ lens and a pyroelectric scanning slit beam profiler was used to measure the transverse beam profiles through the focus.
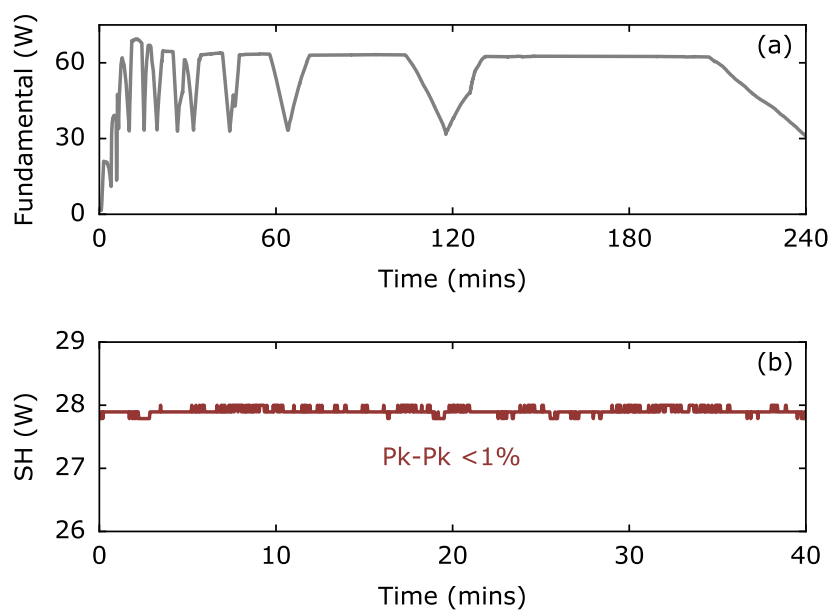

Fig. 3. (a) Polarized fundamental power as a function of time from turn-on, demonstrating oscillations in the degree of polarization. (b) Power stability of the second-harmonic $(\mathrm{SH})$ over a 40 minute period without adjustment of the fundamental polarization.

Figure 4 shows the measured beam diameters (points) with a Gaussian fit to the beam caustic (lines), which revealed an $\mathrm{M}^{2}=1.15$ and $\mathrm{M}^{2}=1.14$ in the vertical and horizontal axes, respectively. A representative image of the collimated SHG beam is shown in the inset of Fig. 4, which confirmed a circular Gaussian beam profile.

The optical spectrum of the generated $\mathrm{SH}$ had a $3 \mathrm{~dB}$ spectral bandwidth of $0.10 \mathrm{~nm}$ (red line Fig. 5), measured using an optical spectrum analyzer with a $10 \mathrm{pm}$ resolution bandwidth (Advantest Q8384). The SH spectrum displayed low-level modulations caused by the sinc ${ }^{2}$ spectral acceptance function of the PPLN crystal. These modulations were visible due to the existence of a pedestal in the optical spectrum of the fundamental (black line Fig. 5), which was caused by pump-degenerate four-wave mixing in the Er:fiber MOPA. The $3 \mathrm{~dB}$ spectral bandwidth of the fundamental was $0.20 \mathrm{~nm}$, therefore, not all of the power was contained within the spectral acceptance bandwidth of the PPLN crystal $(0.41 \mathrm{~nm})$. It is expected that higher SHG conversion efficiencies would be obtained with a narrower linewidth pump laser.

\section{CONCLUSION}

In conclusion, we have demonstrated the highest reported CW average-power generated by SHG using a periodically poled crystal. An average-power of $28.2 \mathrm{~W}$ at $780 \mathrm{~nm}$ was generated with a conversion efficiency of $45 \%$, with near diffraction-limited beam quality $\left(\mathrm{M}^{2} \leq 1.15\right)$ using a PPLN crystal in a simple, single-pass setup. The second-harmonic had a $3 \mathrm{~dB}$ spectral bandwidth of $0.10 \mathrm{~nm}$. The $\mathrm{SHG}$ conversion efficiency monotonically increased for all fundamental powers used, suggesting that the SH average-power can be further scaled by using a more powerful pump laser. The demonstrated architecture is a practical and effective route to generating high brightness radiation that is simpler to implement in comparison to power scaling existing laser systems operating around $780 \mathrm{~nm}$. 


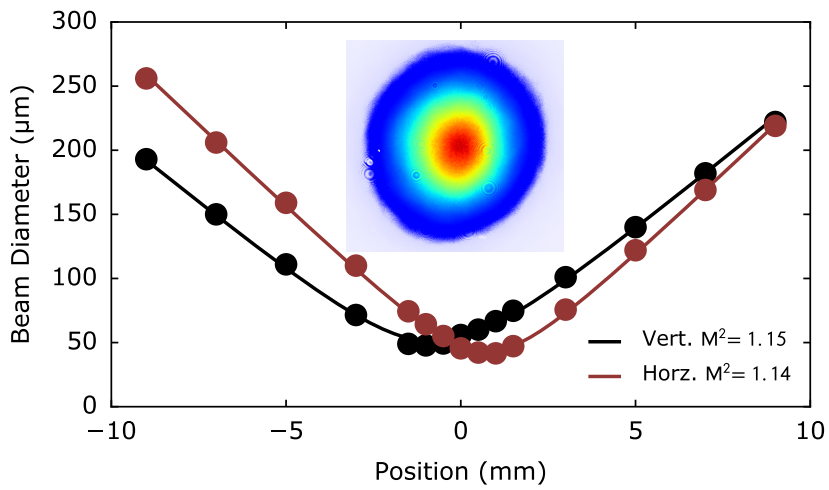

Fig. 4. Beam diameter (points) of the generated $780 \mathrm{~nm}$ at full power through the focus of an $\mathrm{f}=120 \mathrm{~mm}$ lens in the horizontal and vertical axes with Gaussian beam caustic fits (lines). Inset: typical CCD image of the collimated beam profile.

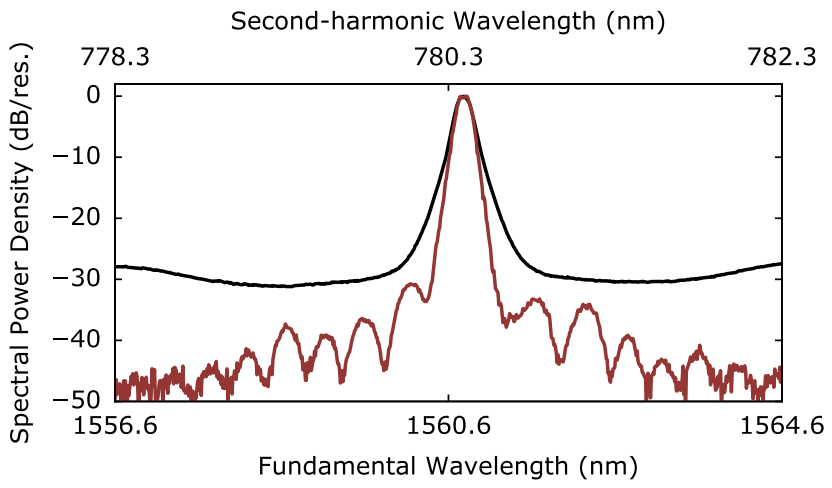

Fig. 5. Optical spectrum of the fundamental (black) and generated secondharmonic (red).

\section{ACKNOWLEDGMENT}

The authors acknowledge the support of IPG Photonics for much of the equipment used in the experiments presented herein. We also thank R. I. Woodward and E. J. R. Kelleher for engaging discussions. The data used in this paper is openly available at: http://doi.org/10.5281/zenodo.803307.

\section{REFERENCES}

[1] S. S. Sané, S. Bennetts, J. E. Debs, C. C. N. Kuhn, G. D. McDonald, P. A. Altin, J. D. Close, and N. P. Robins, " 11 W narrow linewidth laser source at $780 \mathrm{~nm}$ for laser cooling and manipulation of Rubidium," Opt. Express 20(8), 8915-8919 (2012).

[2] W. F. Krupke, R. J. Beach, V. K. Kanz, and S. A. Payne, "Resonance transition 795-nm rubidium laser," Opt. Lett. 28(23), 2336-2338 (2003).

[3] B. Sumpf, K. H. Hasler, P. Adamiec, F. Bugge, F. Dittmar, J. Fricke, H. Wenzel, M. Zorn, G. Erbert and G. Tränkle, "High-Brightness Quantum Well Tapered Lasers," IEEE J. Sel. Top. Quant. 15(15), 1009-1020 (2009).

[4] G. Erbert, I. Bass, R. Hackel, S. Jenkins, K. Kanz, and J. Paisner, "43W, cw Ti:sapphire laser," in Conference on Lasers and Electro-Optics, 1991 OSA Technical Digest Series (Optical Society of America, 1991), paper CThH4.

[5] K. Gürel, V. J. Wittwer, M. Hoffmann, C. J. Saraceno, S. Hakobyan, B. Resan, A. Rohrbacher, K. Weingarten, S. Schilt, and T. Südmeyer, "Green-diode-pumped femtosecond Ti:Sapphire laser with up to 450 mW average power," Opt. Express 23(23), 30043-30048 (2015).
[6] U. Demirbas and I. Baali, "Power and efficiency scaling of diode pumped Cr:LiSAF lasers: $770-1110 \mathrm{~nm}$ tuning range and frequency doubling to 387-463 nm," Opt. Lett. 40(20), 4615-4618 (2015).

[7] U. Demirbas, I. Baali, D. A. E. Acar, and A. Leitenstorfer, "Diodepumped continuous-wave and femtosecond Cr:LiCAF lasers with high average power in the near infrared, visible and near ultraviolet," Opt. Express 23(7), 8901-8909 (2015).

[8] A. Teppitaksak, A. Minassian, G. M. Thomas, and M. J. Damzen, "High efficiency $>26 \mathrm{~W}$ diode end-pumped Alexandrite laser," Opt. Express 22(13), 16386-16392 (2014).

[9] E. A. Arbabzadah and M. J. Damzen, "Fibre-coupled red diode-pumped Alexandrite $\mathrm{TEM}_{00}$ laser with single and double-pass end-pumping," Laser Phys. Lett. 13(6), 065002-065008 (2016).

[10] Y. Jeong, S. Yoo, C. A. Codemard, J. Nilsson, J. K. Sahu, D. N. Payne, R. Horley, P. W. Turner, L. Hickey, A. Harker, M. Lovelady, and A. Piper "Erbium:Ytterbium Codoped Large-Core Fiber Laser With 297W Continuous-Wave Output Power," IEEE J. Sel. Top. Quant. 13(3), 573-579, (2007)

[11] D. N. Nikogosyan, Nonlinear Optical Crystals: A Complete Survey (Springer, 2005).

[12] S. Chiow, T. Kovachy, J. M. Hogan, and M. A. Kasevich, "Generation of $43 \mathrm{~W}$ of quasi-continuous $780 \mathrm{~nm}$ laser light via high-efficiency, singlepass frequency doubling in periodically poled lithium niobate crystals," Opt. Lett. 37(18), 3861-3863 (2012).

[13] M. Y. Vyatkon, A. V. Avdokhin, A. G. Dronov, R. I. Yagodkin, S. V. Popov, J. R. Taylor, and V. P. Gapontsev, "Operation Limits of FluxGrown PPKTP and Stoichiometric PPLT for High Power SHG around 775nm," in Advanced Solid-State Photonics, Technical Digest (Optical Society of America, 2005), paperTuB25.

[14] S. Sinha, D. S. Hum, K. E. Urbanek, Y. Lee, M. J. F. Digonnet, M M. Fejer, and R. L. Byer, "Room-Temperature Stable Generation of 19 Watts of Single-Frequency 532-nm Radiation in a Periodically Poled Lithium Tantalate Crystal," J. Lightwave Technol. 26(24), 3866-3871 (2008).

[15] H. H. Lim, T. Katagai, S. Kurimura, T. Shimizu, K. Noguchi, N. Ohmae, N. Mio, and I. Shoji, "Thermal performance in high power SHG characterized by phase-matched calorimetry,” Opt. Express 19(23), 22588-22593 (2011).

[16] D. White, E. Dawes, and J. Marburger, "Theory of second-harmonic generation with high-conversion efficiency," IEEE J. Quantum Elect. 6(12), 793-796 (1970).

[17] G. D. Boyd, and D. A. Kleinman, "Parametric Interaction of Focused Gaussian Light Beams," J. Appl. Phys. 39(8), 3597-3639 (1968).

[18] O. A. Louchev, N. E. Yu, S. Kurimura, and K. Kitamura, "Thermal inhibition of high-power second-harmonic generation in periodically poled $\mathrm{LiNbO}_{3}$ and $\mathrm{LiTaO}_{3}$ crystals," Appl. Phys. Lett. 87(13), 131101131103 (2005). 